Z. Epileptol. 2021 · 34:390-395 https://doi.org/10.1007/s10309-021-00443-y Angenommen: 24. August 2021 Online publiziert: 30 . September 2021 ๑) Der/die Autor(en) 2021

\section{Akut symptomatische epileptische Anfälle in Assoziation mit COVID-19}

\author{
Matthias Mauritz ${ }^{1,3}$ Eugen Trinka ${ }^{1,2,3}$ \\ 'Universitätsklinik für Neurologie, Christian Doppler Klinik, Paracelsus Medizinische Universität und \\ Centre for Cognitive Neuroscience, Salzburg, Österreich \\ ${ }^{2}$ Department of Public Health, Health Services Research and Health Technology Assessment, UMIT - \\ University for Health Sciences, Medical Informatics and Technology, Hall in Tirol, Österreich \\ ${ }^{3}$ Neuroscience Institute, Christian Doppler Klinik, Paracelsus Medizinische Universität und Centre for \\ Cognitive Neuroscience, Salzburg, Österreich
}

\section{Zusammenfassung}

\section{In diesem Beitrag}

- Neurotropismus von SARS-CoV-2

- Neurologische Manifestationen von COVID-19

- Akut symptomatische epileptische Anfälle in Assoziation mit COVID-19

- Neuropädiatrische Aspekte bei COVID-19

- Behandlung von akut symptomatischen epileptischen Anfällen bei COVID-19

- Epileptische Anfälle und COVID-19Impfungen

- Zusammenfassung

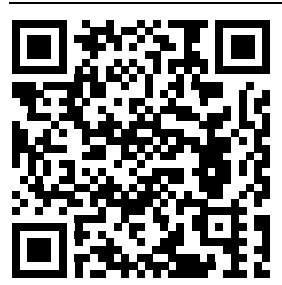

QR-Code scannen \& Beitrag online lesen

Akut symptomatische epileptische Anfälle treten in einem engen zeitlichen Zusammenhang zu einer akuten strukturellen oder funktionellen Schädigung des Gehirns auf, die viele verschiedene Ursachen haben kann. Neurologische Symptome wie Enzephalopathie, Geruchsstörung und Kopfschmerzen finden sich häufig bei COVID-19. Epileptische Anfälle ereignen sich nur in 1-2\% aller mit COVID19 hospitalisierten Patient*innen. Eine mögliche direkte Invasion des ZNS durch SARS-CoV-2 sowie indirekte Effekte durch Hypoxie, Inflammation und metabolische Entgleisungen werden als Ursache für die neurologischen Manifestationen bei COVID19 angenommen.

\section{Schlüsselwörter}

Pandemie - Epilepsie - Enzephalopathie · SARS-Cov-2
Im Dezember 2019 wurde in Wuhan, China, das Auftreten eines Clusters von Patient*innen mit interstitiellen Pneumonien unbekannter Ursache berichtet. Anfang Januar 2020 wurde als Pathogen ein neues Betacoronavirus identifiziert und aufgrund der phylogenetischen Ähnlichkeit mit dem Erreger des Severe Acute Respiratory Syndrome (SARS) als Severe Acute Respiratory Syndrome Coronavirus 2 (SARS-CoV-2) bezeichnet [48]. Die von SARS-CoV-2 ausgelöste Erkrankung wird Coronavirus Disease 2019 (COVID-19) genannt. Bislang sind Stand 16.05.2021 weltweit rund 162 Mio. Menschen an COVID-19 erkrankt und 3,3 Mio. Menschen an den direkten Folgen der Erkrankung verstorben [46].

\section{Neurotropismus von SARS-CoV-2}

Bereits kurz nach dem Beginn der COVID19-Pandemie wurden erste Berichte über das Auftreten von neurologischen Mani- festationen im Rahmen von COVID-19 Erkrankungen veröffentlicht. Eine initiale retrospektive Studie von 214 mit bestätigter SARS-CoV-2-Infektion hospitalisierten Patient*innen aus Wuhan, China, beschrieb das Auftreten von neurologischen Symptomen in 36,4\% [32]. Einzelne Fallberichte von Enzephalitis in Assoziation mit SARSCoV-2-Infektionen als mögliche Manifestation einer direkten ZNS-Invasion des Virus wurden veröffentlicht $[5,35,38]$. Der Nachweis von SARS-CoV-2-RNA mittels PCR aus dem Liquor gelang aber nur selten, und die klinische Evidenz für direkt durch SARSCoV-2 vermittelte Enzephalitiden ist bislang gering. Es lagen bislang aus Untersuchungen zu den saisonalen Coronaviren und SARS-CoV/MERS-CoV experimentelle und klinische Evidenz für das Potenzial von Coronaviren, das Nervensystem von Menschen zu befallen, vor [6]. Es existieren Fallberichte von Enzephalitis, akut disseminierter Enzephalomyelitis (ADEM), Schlaganfällen und verschiede- 
nen neuromuskulären Manifestationen bei Patient*innen mit SARS und MERS [14]. Auch bei anderen respiratorischen Virusinfektionen wie Influenza wurden neurologische Komplikationen wie Enzephalopathie und Enzephalitis, epileptische Anfälle oder Guillain-Barré-Syndrome berichtet [36]. Es ist bekannt, dass der ACE2-Rezeptor, durch dessen Bindung SARS-CoV und SARS-CoV-2 menschliche Zellen infizieren, an verschiedensten Stellen im ZNS, besonders auf Neuronen im Hirnstamm sowie auf Endothelzellen exprimiert wird [31]. Als mögliche Routen einer direkten Invasion von SARS-CoV-2 in das ZNS wurden unter anderem die Infektion von olfaktorischen Neuronen im Riechepithel und der konsekutive retrograde axonale Transport des Virus in das ZNS, die Überwindung der Blut-Hirn-Schranke im Rahmen einer Virämie und/oder durch Infektion von Endothelzellen und der Transport des Virus in das ZNS durch infizierte Leukozyten postuliert [14, 37]. Bisherige Ergebnisse aus neuropathologischen Studien und Liquoruntersuchungen zu SARS-CoV-2 stellen dessen Virulenz für das Nervensystem teilweise infrage: So konnte beispielsweise die RNA des Virus in den Zellen der Riechschleimhaut, nicht aber eindeutig in den olfaktorischen Neuronen nachgewiesen werden [20,33]. Zudem wird der ACE2Rezeptor von Zellen der Riechschleimhaut, nicht aber von olfaktorischen Neuronen exprimiert [7]. Mehrere Autopsieserien konnten SARS-CoV-2 entweder nicht oder nur in geringen Mengen im Gehirn nachweisen [42]. Ein systematischer Review zu Liquoranalysen bei Patient*innen mit COVID-19 und neurologischen Symptomen fand positive SARS-CoV-2-PCR-Analysen in nur 17 von 304 (6\%) Fällen [29]. In manchen Fällen wurden hohe Cycle-threshold-Werte angegeben, sodass mögliche Kontaminationen vermutet wurden [20]. Derzeit werden als Ursache für die meisten neurologischen Manifestationen in Assoziation mit COVID19 überwiegend indirekte Mechanismen und Auswirkungen der systemischen Erkrankung auf das Gehirn wie metabolische Entgleisungen (z.B. Hyponatriämie, Urämie), Hypoxie, Inflammation, Hyperkoagulopathie und endotheliale Dysfunktion angenommen [20].

\section{Neurologische Manifestationen von COVID-19}

Neurologische Symptome und Manifestationen werden bei Patient*innen mit COVID-19 in verschiedenen Arbeiten in unterschiedlicher Häufigkeit, je nach Studie zwischen 3,5-84\%, gefunden [21, 47]. Die berichteten neurologischen Manifestationen umfassen Enzephalopathie, Geruchs- und Geschmacksstörung, Kopfschmerzen, zerebrovaskuläre Erkrankungen wie ischämischer Schlaganfall, intrazerebrale Blutungen und zerebrale Sinusvenenthrombosen, epileptische Anfälle, hypoxische Hirnschädigung sowie para-/postinfektiöse Syndrome wie Guillain-Barré-Syndrom, akute disseminierte Enzephalomyelitis (ADEM) und akute nekrotisierende Enzephalopathie [20]. Eine in mehreren Krankenhäusern in New York City über 2 Monate durchgeführte, prospektive Beobachtungsstudie von 4491 mit COVID-19 hospitalisierten Patient*innen fand ein Auftreten von neuen neurologischen Manifestationen in 14\% [17]. Die ersten neurologischen Symptome traten im Median 2 Tage nach den ersten COVID-19-Symptomen auf. Als häufigste neue neurologische Diagnose wurde eine metabolische Enzephalopathie festgestellt $(6,8 \%)$, gefolgt von Schlaganfällen $(1,9 \%)$, epileptischen Anfällen (1,6\%) und hypoxischer Enzephalopathie (1,4\%). Es wurden in dieser Arbeit keine Fälle von Meningitis, Enzephalitis oder Myelitis in Verbindung mit SARS-CoV-2 identifiziert. Patient*innen, bei denen eine neue neurologische Manifestation auftrat, waren schwerer von COVID-19 betroffen, was sich z. B. an einer signifikant höheren Rate an invasiver Beatmung oder an einem längeren Spitalaufenthalt zeigte. Zudem hatten Patient*innen mit neuen neurologischen Erkrankungen in dieser Arbeit eine signifikant höhere Mortalität gegenüber Patient*innen mit COVID-19 ohne neurologische Manifestationen [17]. Neben den akuten neurologischen Komplikationen werden neurokognitive Beeinträchtigungen wie mentale Erschöpfung, Gedächtnisstörungen und dysexekutives Syndrom als Langzeitfolgen einer COVID19-Erkrankung berichtet [34, 40].

\section{Akut symptomatische epileptische Anfälle in Assoziation mit COVID-19}

In der bislang größten Untersuchung zu neurologischen Manifestationen bei COVID-19 von Frontera et al. fanden sich epileptische Anfälle bei 74 von $4491 \mathrm{~Pa}$ tient*innen $(1,6 \%)$ und stellten damit nach Enzephalopathie und Schlaganfällen die dritthäufigste neurologische Manifestation dar. Bei 34 der 74 Patient*innen (46\%) war bislang keine Diagnose einer Epilepsieerkrankung bekannt gewesen [17]. In einer systematischen Übersichtsarbeit der Literatur zu neurologischen Manifestationen bei COVID-19 wurden epileptische Anfälle etwas seltener in $0,7 \%$ der insgesamt 68.361 untersuchten Fälle angegeben [8]. Das Auftreten von epileptischen Anfällen bei Patient*innen mit COVID-19 wurde auch in mehreren Fallberichten und Fallserien beschrieben [3, 22].

Epileptische Anfälle können das Symptom einer COVID-19-Erkrankung sein, das zur Erstvorstellung der Patient*innen in einer Notaufnahme führt. In einer Untersuchung von allen über einen Zeitraum von 2 Wochen hospitalisierten COVID-19Patient*innen im Iran, hatte in 45 von 5872 Fällen $(0,8 \%)$ ein epileptischer Anfall zur Aufnahme in das Krankenhaus geführt [24]. Nur 9\% dieser COVID-19-Patient*innen mit epileptischen Anfällen hatten eine Anamnese von Epilepsie. Ähnliche Ergebnisse fanden sich bei 1043 mit COVID-19 über einen Zeitraum von 6 Wochen hospitalisierten Patient*innen in Boston, USA. In dieser Kohorte war bei $7 \mathrm{~Pa}$ tient*innen (0,8\%) ein epileptischer Anfall das präsentierende Symptom bei Krankenhausaufnahme gewesen [2]. Davon hatten 3 Patient*innen keinerlei andere COVID19-Symptome wie Husten oder Fieber vor dem Anfall gezeigt. Bei 3 Patient*innen war eine Epilepsieerkrankung bekannt gewesen, in den 4 anderen Fällen fand sich bei 2 Patient*innen ein Schlaganfall in der Anamnese. Bei 3 Patient*innen fanden sich schwere metabolische Entgleisungen in Form von Hyponatriämie, Urämie und dialysepflichtigem Nierenversagen.

Epileptische Anfälle treten bei 15-20\% aller kritisch kranken Patient*innen auf Intensivstationen auf. Die meisten dieser 
Anfälle zeigen keine klinisch erkennbaren Zeichen und können nur mittels Anwendung einer kontinuierlichen EEG-Ableitung (",continuous EEG monitoring " [CEEG]) detektiert werden [12]. Zwei unterschiedliche Arbeiten, bei denen bei jeweils 22 COVID-19-Patient*innen aufgrund von Enzephalopathie oder vorausgegangenen klinischen Anfallsereignissen eine cEEGAbleitung durchgeführt wurde, fanden bei allen Patient*innen eine Verlangsamung der Hintergrundaktivität. Bei jeweils rund einem Drittel der Patientin*innen fanden sich epileptiforme Potenziale [18, 30]. Zwei Patient*innen mit elektroenzephalographischen Anfällen, die keine Anamnese einer Epilepsieerkrankung hatten und bei denen eine Bildgebung des Gehirns keine akute strukturelle zerebrale Pathologie gezeigt hatte, wurden berichtet. In einer weiteren cEEG-Studie von 111 COVID19-Patient*innen, von denen sich rund drei Viertel zum Zeitpunkt der EEG-Ableitung auf der Intensivstation befanden und eine schwere Bewusstseinsstörung aufwiesen, fanden sich bei $30 \%$ epileptiforme Potenziale und bei $7 \%$ epileptische Anfälle [39]. Bei 4\% der Patient*innen wurden ausschließlich nichtkonvulsive bzw. elektroenzephalographische Anfälle beobachtet.

Status epilepticus (SE) bei Patient*innen mit COVID-19 wurde ebenfalls in Fallberichten beschrieben [1, 10, 45]. Ein systematischer Review zu SE und COVID19 fand 47 Fälle eines SE in Assoziation mit einer SARS-CoV-2-Infektion [13]. Nur 3 Patient*innen in dieser Kohorte hatten eine bekannte Epilepsieerkrankung. Für die meisten SE-Fälle wurde eine akut symptomatische Ursache des SE angenommen, allerdings konnte die Ätiologie des SE in $55 \%$ nicht identifiziert werden. In 14,9\% der Fälle wurde die Ätiologie des SE als „vaskulär" (intrazerebrale Blutung, posteriores reversibles Enzephalopathiesyndrom [PRES], ischämischer Schlaganfall) beurteilt, gefolgt von "septisch" in $10,6 \%$ und "inflammatorisch" in 8,6\%. Bei 4 Patient*innen mit SE wurde eine positive SARS-CoV-2-PCR aus dem Liquor berichtet. Bei der Mehrheit der Patient*innen trat der SE nach Beginn der COVID-19Symptome auf. Die meisten Patient*innen in dieser Arbeit hatten einen konvulsiven SE mit prominenten motorischen Symp- tomen. Die relativ geringe Repräsentation des nichtkonvulsiven SE (NCSE) in dieser Population und die Tatsache, dass in nur $70 \%$ der in dieser Arbeit untersuchten SE-Patient*innen ein EEG durchgeführt wurde, gaben den Autoren Anlass zur Vermutung, dass NCSE unter anderem aufgrund von Limitationen in der EEGDiagnostik bei infektiösen Patient*innen unterdiagnostiziert sein könnte [13, 19]. Eine epidemiologische Untersuchung aus Salzburg, Österreich, konnte keinen Unterschied in der Inzidenz des SE zwischen den Monaten der COVID-Pandemie März und April 2020, in denen in Österreich strikte Lockdown-Maßnahmen herrschten, und demselben Zeitraum in den Jahren 2018 und 2019 feststellen [28]. Es fand sich ein nichtsignifikanter Trend zu weniger Diagnosen von NCSE im Zeitraum der COVID-Pandemie in dieser Arbeit.

\section{Neuropädiatrische Aspekte bei COVID-19}

Kinder aller Altersgruppen können von einer SARS-CoV-2-Infektion und COVID-19 betroffen sein [11]. Infektionen mit SARSCoV-2 sind bei Kindern wahrscheinlich genauso häufig wie bei Erwachsenen, allerdings entwickeln Kinder, besonders jene unter 10 Jahren, deutlich seltener COVID19-Symptome oder einen schweren Krankheitsverlauf [49]. In einer retrospektiven Untersuchung von 1695 Patient*innen, die jünger als 21 Jahre waren und aufgrund einer schweren COVID-19-Erkrankung oder MIS-C hospitalisiert waren, fanden sich bei $22 \%$ neurologische Symptome [27]. Von diesen wiederum hatten $12 \%$ eine schwerwiegende neurologische Manifestation. Unter anderem wurden schwere Enzephalopathien, Schlaganfälle, Enzephalitis und demyelinisierende ZNS-Erkrankungen sowie Fälle von Guillain-Barré-Syndrom beobachtet.

Fieberkrämpfe sind besonders mit viralen Infektionen wie Influenza und humanes Herpesvirus Typ 6, die hohes Fieber verursachen, assoziiert, aber treten auch in Zusammenhang mit Infektionen durch saisonale Coronaviren auf [9]. Eine Arbeit aus Rom, Italien, berichtete für den Zeitraum der COVID-19-Pandemie von März bis Mai 2020 eine signifikant höhere Zahl an Krankenhausaufnahmen aufgrund von
Fieberkrämpfen im Vergleich zum Vorjahr [41]. Von 25 Kindern mit Fieberkrämpfen konnte allerdings nur bei 2 Kindern SARSCoV-2 mittels PCR aus dem Nasopharyngealabstrich nachgewiesen werden. Insgesamt sind die Hinweise für eine relevante Rolle von SARS-CoV-2 für das Auftreten von epileptischen Anfällen (insbesondere Fieberkrämpfen) bei Kindern bislang gering.

\section{Behandlung von akut symptomatischen epileptischen Anfällen bei COVID-19}

Eine erfolgreiche Behandlung von Patient*innen mit akut symptomatischen epileptischen Anfällen setzt voraus, dass diese als solche erkannt werden und die zugrunde liegende Ätiologie rasch identifiziert wird, um eine mögliche kausale Therapie frühzeitig etablieren zu können (z.B. mechanische Thrombektomie bei ischämischem Schlaganfall oder therapeutische Heparinisierung bei zerebraler Sinusvenenthrombose). Daneben werden Patient*innen mit akut symptomatischen Anfällen in der Regel vorübergehend mit Anfallsmedikamenten behandelt, um das Risiko für weitere Anfälle in der akuten Phase der zugrundeliegenden ZNS-Erkrankung zu reduzieren. In Zusammenhang mit COVID-19 wurde auf das mögliche Potenzial von pharmakokinetischen Interaktionen zwischen Anfallsmedikamenten und COVID-19-Therapien hingewiesen [16]. Beispielsweise können hepatische Enzyminduktoren wie Carbamazepin und Phenytoin die Konzentration von Remdesivir, das häufig in der Behandlung von schwer kranken COVID-19-Patient*innen eingesetzt wird, signifikant reduzieren [26]. Von der Universität Liverpool wird ein frei verfügbares Online-Tool zum Interaktionscheck von COVID-19-Therapien mit anderen Pharmaka angeboten [43].

\section{Epileptische Anfälle und COVID- 19-Impfungen}

Zum derzeitigen Stand (Mai 2021) sind 4 verschiedene Impfstoffe zur Prävention einer SARS-CoV-2-Infektion bzw. des Auftretens einer COVID-19-Erkrankung in der Europäischen Union zugelassen [15]. Die Internationale Liga gegen Epilepsie (ILAE) 
hat in einer Stellungnahme festgehalten, dass derzeit kein erhöhtes Risiko für das Auftreten von epileptischen Anfällen als Nebenwirkung von COVID-19-Impfungen bekannt ist [23]. Als Impfreaktion kann es zum Auftreten von Fieber kommen, was die "Krampfschwelle" („,seizure threshold“) herabsetzen kann. Im Zusammenhang mit Anfallsmedikation und COVID-19-Impfungen wurde darauf hingewiesen, dass bekannt ist, dass es nach Influenzaimpfungen zu durch Zytokine vermittelten Änderungen in der Expression von hepatischen $\mathrm{Cy}$ tochrom-P450-Enzymen kommt und dadurch die Konzentration von Anfallsmedikamenten (z. B. Carbamazepin) beeinflusst werden kann [25]. Zu möglichen Interaktionen zwischen COVID-19-Impfungen und antiepileptischer Medikation liegen derzeit keine Informationen vor.

\section{Zusammenfassung}

Es lässt sich zu den bisher vorliegenden Daten zu epileptischen Anfällen bei COVID19 festhalten, dass epileptische Anfälle bei Patient*innen mit COVID-19 eine relativ seltene, aber wichtige neurologische Manifestation darstellen können. Ein Großteil der bisher berichteten Anfälle trat bei Patient*innen ohne Anamnese einer Epilepsieerkrankung auf. Für diese Anfälle dürfte ein akut symptomatischer Zusammenhang zur COVID-19-Erkrankung möglich bis wahrscheinlich zu sein. Bei einigen der berichteten Anfallsereignisse könnte es sich aber auch um unprovozierte epileptische Anfälle gehandelt haben, die nur in simpler Koinzidenz zur SARS-CoV-2-Infektion aufgetreten sind [44]. Zu beachten ist, dass es den derzeit vorliegenden Informationen zu Anfällen bei COVID-19 zum Teil an Details fehlt bzw. die zugrunde liegenden Arbeiten deutliche methodische Limitationen aufweisen. So wird in manchen Arbeiten nicht berichtet, ob Patient*innen mit Anfällen eine Anamnese von früheren Anfallsereignissen oder Epilepsie haben. In vielen Fällen fehlen Informationen zum diagnostischen Work-up, was die Beurteilung und ätiologische Zuordnung der berichteten Anfälle erschwert. Eine durch direkte Effekte des Virus auf das ZNS vermittelte Ätiologie der akut symptomatischen epileptischen Anfälle wie z. B. im Rahmen einer Enzephalitis scheint eher die Ausnahme zu sein. Das Zusammenspiel von multiplen Faktoren wie Hypoxie, Sepsis, Inflammation sowie schweren metabolischen Entgleisungen wie Hyponatriämie und Urämie, die sich bei COVID-19 häufig finden, könnte bei kritisch kranken Patient*innen das Auftreten von epileptischen Anfällen verursachen [20]. Zudem ist COVID-19, wie oben beschrieben, mit dem Auftreten mehrerer Erkrankungen des Gehirns assoziiert, für die wiederum selbst ein Zusammenhang mit akut symptomatischen epileptischen Anfällen bekannt ist. So treten akut symptomatische Anfälle bei 2-4\% aller Patient*innen mit ischämischem Schlaganfall und 10-18\% aller Patient*innen mit einer intrazerebralen Blutung auf [4]. Aus den bislang vorliegenden Informationen lässt sich vermuten, dass epileptische Anfälle eher bei Patient*innen auftreten, die schwerer von COVID-19 betroffen sind. Die Detektion von Anfällen kann bei kritisch kranken Patient*innen mit COVID-19, die invasiv beatmet werden und muskelrelaxiert sind oder eine Enzephalopathie/Bewusstseinsstörung aufweisen, unmöglich sein, sofern keine kontinuierliche EEG-Ableitung angewandt wird.

\section{Fazit für die Praxis}

Epileptische Anfälle finden sich bei 1-2\% der Patient*innen mit COVID-19. COVID-19 kann zudem mit verschiedenen anderen neurologischen Komplikationen assoziiert sein, als deren Symptom epileptische Anfälle auftreten. Bei Patient*innen mit COVID-19 und epileptischen Anfällen sollte daher unbedingt weitere Diagnostik (z. B. zerebrale Bildgebung) erfolgen, um mögliche kausal behandelbare Ursachen rasch zu erkennen. Bei COVID-19-Patient*innen mit Bewusstseinsstörung und dem klinischen Verdacht auf das Vorliegen von epileptischen Anfällen sollte eine kontinuierliche EEG Ableitung durchgeführt werden. Vor der Anwendung von Anfallsmedikation sollte an die Möglichkeit von Interaktionen mit COVID-19-Therapien gedacht und ein Interaktionscheck durchgeführt werden.

\section{Korrespondenzadresse}

Univ.-Prof. Dr. Mag. Eugen Trinka, FRCP Universitätsklinik für Neurologie, Christian Doppler Klinik, Paracelsus Medizinische Universität und Centre for Cognitive Neuroscience

Ignaz-Harrer-Str. 79, 5020 Salzburg, Österreich e.trinka@salk.at

Funding. Open access funding provided by Paracelsus Medical University.

\section{Einhaltung ethischer Richtlinien}

Interessenkonflikt. E. Trinka erhält unabhängig von dieser Arbeit Honorare von EVER Pharma, Marinus, Arvelle, Argenix, Medtronic, Bial-Portela \& Ca, NewBridge, GL Pharma, GlaxoSmithKline, Boehringer Ingelheim, LivaNova, Eisai, UCB, Biogen, Genzyme Sanofi und Actavis; UCB Pharma, Eisai, Red Bull, Merck, Bayer, the European Union, FWF - Österreichischer Fond zur Wissenschaftsförderung und dem Bundesministerium für Wissenschaft und Forschung und Jubiläumsfond der Österreichischen Nationalbank. M. Mauritz gibt an, dass kein Interessenkonflikt besteht.

Für diesen Beitrag wurden von den Autoren keine Studien an Menschen oder Tieren durchgeführt. Für die aufgeführten Studien gelten die jeweils dort angegebenen ethischen Richtlinien.

Open Access. Dieser Artikel wird unter der Creative Commons Namensnennung 4.0 International Lizenz veröffentlicht, welche die Nutzung, Vervielfältigung, Bearbeitung, Verbreitung und Wiedergabe in jeglichem Medium und Format erlaubt, sofern Sie den/die ursprünglichen Autor(en) und die Quelle ordnungsgemäß nennen, einen Link zur Creative Commons Lizenz beifügen und angeben, ob Änderungen vorgenommen wurden.

Die in diesem Artikel enthaltenen Bilder und sonstiges Drittmaterial unterliegen ebenfalls der genannten Creative Commons Lizenz, sofern sich aus der Abbildungslegende nichts anderes ergibt. Sofern das betreffende Material nicht unter der genannten Creative Commons Lizenz steht und die betreffende Handlung nicht nach gesetzlichen Vorschriften erlaubt ist, ist für die oben aufgeführten Weiterverwendungen des Materials die Einwilligung des jeweiligen Rechteinhabers einzuholen.

Weitere Details zur Lizenz entnehmen Sie bitte der Lizenzinformation auf http://creativecommons.org/ licenses/by/4.0/deed.de.

\section{Literatur}

1. Abdulsalam MA, Abdulsalam AJ, Shehab D (2020) Generalized status epilepticus as a possible manifestation of COVID-19. Acta Neurol Scand 142(4):297-298

2. Anand P, Al-Faraj A, Sader E, Dashkoff J, Abdennadher M, Murugesan R et al (2020) Seizure as the presenting symptom of COVID-19: a retrospective case series. Epilepsy Behav 112:107335 
3. Asadi-Pooya AA (2020) Seizures associated with coronavirus infections. Seizure 79:49-52

4. Beghi E, D'Alessandro R, Beretta $S$, Consoli D, Crespi V, Delaj L et al (2011) Incidence and predictors of acute symptomatic seizures after stroke. Neurology 77(20):1785-1793

5. Bernard-Valnet R, Pizzarotti B, Anichini A, Demars Y, Russo E, Schmidhauser Metal (2020) Two patients with acutemeningoencephalitisconcomitant with SARS-CoV-2 infection. Eur J Neurol 27(9):e43-e4

6. Bohmwald K, Gálvez NMS, Ríos M, Kalergis AM (2018) Neurologic alterations due to respiratory virus infections. Front Cell Neurosci 12:386

7. Brann DH, Tsukahara T, Weinreb C, Lipovsek M Van den Berge K, Gong B et al (2020) Nonneuronal expression of SARS-CoV-2 entry genes in the olfactory system suggests mechanisms underlying COVID-19-associated anosmia. Sci Adv 6(31):eabc5801. https://doi.org/10.1126/sciadv. abc5801

8. Cagnazzo F, Arquizan C, Derraz I, Dargazanli C, Lefevre PH, Riquelme $C$ et al (2020) Neurological manifestations of patients infected with the SARS-CoV-2: a systematic review of the literature. J Neurol. https://doi.org/10.1007/s00415-02010285-9

9. Camfield P, Camfield C (2015) Febrile seizures and genetic epilepsy with febrile seizures plus (GEFS+). Epileptic Disord 17(2):124-133

10. Carroll E, Neumann H, Aguero-Rosenfeld ME, Lighter J, Czeisler BM, Melmed K et al (2020) Post-COVID-19 inflammatory syndrome manifesting as refractory status epilepticus. Epilepsia 61(10):e135-e9

11. Christy A (2020) COVID-19: a review for the pediatric neurologist.J Child Neurol 35(13):934-939

12. Claassen J, Mayer SA, Kowalski RG, Emerson RG, Hirsch LJ (2004) Detection of electrographic seizures with continuous EEG monitoring in critically ill patients. Neurology 62(10):1743-1748

13. Dono F, Nucera B, Lanzone J, Evangelista G, Rinaldi F, Speranza R et al (2021) Status epilepticus and COVID-19: a systematic review. Epilepsy Behav 118:107887

14. Ellul MA, Benjamin L, Singh B, Lant S, Michael BD, Easton $A$ et al (2020) Neurological associations of COVID-19. Lancet Neurol 19(9):767-783

15. European Medicines Agency (2021) COVID-19 vaccines: authorised. https://www.ema.europa. eu/en/human-regulatory/overview/publichealth-threats/coronavirus-disease-covid-19/ treatments-vaccines/vaccines-covid-19/covid19-vaccines-authorised\#authorised-covid-19vaccines-section.Zugegriffen: 6. Juni 2021

16. Fırat O, Yalçın N, Demirkan K (2020) COVID-19 \& antiepileptic drugs: should we pay attention? Seizure 80:240-241

17. Frontera JA, Sabadia S, Lalchan R, Fang T, Flusty B, Millar-Vernetti $P$ et al (2021) A prospective study of neurologic disorders in hospitalized patients with COVID-19 in New York City. Neurology 96(4):e575-e86

18. Galanopoulou AS, Ferastraoaru V, Correa DJ, Cherian K, Duberstein S, Gursky J et al (2020) EEG findings in acutely ill patients investigated for SARS-CoV-2/COVID-19: a small case series preliminary report. Epilepsia Open 5(2):314-324

19. Gélisse $P$, Rossetti AO, Genton $P$, Crespel A, Kaplan PW (2020) How to carry out and interpret EEG recordings in COVID-19 patients in ICU? Clin Neurophysiol 131(8):2023-2031

20. Hassett CE, Frontera JA (2021) Neurologic aspects of coronavirus disease of 2019 infection. Curr Opin Infect Dis 34(3):217-227

\section{Acute symptomatic epileptic seizures associated with COVID-19}

Acute symptomatic epileptic seizures occur in close temporal relationship with an acute structural or functional damage of the brain, which can have many different causes. Neurological symptoms, such as encephalopathy, anosmia and headache have been frequently reported in patients with COVID-19. Epileptic seizures occur in only $1-2 \%$ of all persons hospitalized with COVID-19. A possible direct effect by invasion of the CNS by SARS-CoV-2 and indirect effects due to hypoxia, inflammation and metabolic derangements, have been discussed as potential causes of the neurological manifestations in COVID-19.

\section{Keywords}

Pandemic - Epilepsy - Encephalopathy · SARS-CoV-2

21. Helms J, Kremer S, Merdji H, Clere-Jehl R, Schenck M, Kummerlen C et al (2020) Neurologic features in severe SARS-CoV-2 infection. N Engl J Med 382(23):2268-2270

22. Hepburn M, Mullaguri N, George $P$, Hantus $S$, Punia V, Bhimraj A et al (2021) Acute symptomatic seizures in critically ill patients with COVID-19: is there anassociation? NeurocritCare 34(1):139-143

23. International League against Epilepsy (2021) COVID-19 vaccines and people with epilepsy. https://www.ilae.org/patient-care/covid-19and-epilepsy/covid-19-vaccines-and-peoplewith-epilepsy\#English.Zugegriffen: 6. Juni 2021

24. Keshavarzi A, Janbabaei G, Kheyrati L, Ghavamabad LH, Asadi-Pooya AA (2021) Seizure is a rare presenting manifestation of COVID-19. Seizure 86:16-18

25. Kow CS, Hasan SS (2021) Potential interactions between COVID-19 vaccines and antiepileptic drugs. Seizure 86:80-81

26. Kuroda N (2021) Epilepsy and COVID-19: updated evidence and narrative review. Epilepsy Behav 116:107785

27. LaRovere KL, Riggs BJ, Poussaint TY, Young CC, Newhams MM, Maamari M et al (2021) Neurologic involvement in children and adolescents hospitalized in the United States for COVID-19 or multisystem inflammatory syndrome. JAMA Neurol 78(5):536-547

28. Leitinger M, Poppert KN, Mauritz M, Rossini F, Zimmermann G, Rohracher A et al (2020) Status epilepticus admissions during the COVID-19 pandemic in Salzburg-A population-based study. Epilepsia 61(12):e198-e203

29. Lewis A, Frontera J, Placantonakis DG, Lighter J, Galetta S, Balcer L et al (2021) Cerebrospinal fluid in COVID-19: a systematic review of the literature. J Neurol Sci 421:117316

30. Louis S, Dhawan A, Newey C, Nair D, Jehi L, Hantus S et al (2020) Continuous electroencephalography characteristics and acute symptomatic seizures in COVID-19 patients. Clin Neurophysiol 131(11):2651-2656

31. Lukiw WJ, Pogue A, Hill JM (2020) SARS-CoV-2 infectivity and neurological targets in the brain. Cell Mol Neurobiol. https://doi.org/10.1007/ s10571-020-00947-7

32. Mao L, Jin H, Wang M, HuY, Chen S, He Q et al (2020) Neurologic manifestations of hospitalized patient with coronavirus disease 2019 in Wuhan, China. JAMA Neurol 77(6):683-690

33. Meinhardt J, Radke J, Dittmayer C, Franz J, Thomas C, Mothes R et al (2021) Olfactory transmucosal SARS-CoV-2 invasion as a port of central nervous system entry in individuals with COVID-19. Nat Neurosci 24(2):168-175

34. Méndez R, Balanzá-Martínez V, Luperdi SC, Estrada I, Latorre A, González-Jiménez P et al (2021) Short-term neuropsychiatric outcomes and quality of life in COVID-19 survivors. J Intern Med. https://doi.org/10.1111/joim.13262

35. Moriguchi T, Harii N, Goto J, Harada D, Sugawara H, Takamino J et al (2020) A first case of meningitis/ encephalitis associated with SARS-Coronavirus-2. Int JInfect Dis 94:55-58

36. Mylonaki E, Harrer A, Pilz G, Stalzer P, Otto F, Trinka E et al (2020) Neurological complications associated with influenza in season 2017/18 in Austria-a retrospective single center study. J Clin Virol 127:104340

37. Nordvig AS, Fong KT, Willey JZ, Thakur KT, Boehme AK, Vargas WS et al (2021) Potential neurologic manifestations of COVID-19. Neurol Clin Pract 11(2):e135-e46

38. Paniz-Mondolfi A, Bryce C, Grimes Z, Gordon RE, Reidy J, Lednicky J et al (2020) Central nervous system involvement by severe acute respiratory syndrome coronavirus-2 (SARS-CoV-2). J Med Virol 92(7):699-702

39. Pellinen J, Carroll E, Friedman D, Boffa M, Dugan P, Friedman DE et al (2020) Continuous EEG findings in patients with COVID-19 infection admitted to a New York academic hospital system. Epilepsia 61(10):2097-2105

40. Riordan P, Stika M, Goldberg J, Drzewiecki M (2020) COVID-19 and clinical neuropsychology: a review of neuropsychological literature on acute and chronic pulmonary disease. Clin Neuropsychol 34(7-8):1480-1497

41. Smarrazzo A, Mariani R, Valentini F, Lombardi MH, Sinibaldi S, Peschiaroli E et al (2021) Threefold increase in admissions for paediatric febrile convulsions during COVID-19 pandemic could indicate alternative virus symptoms. Acta Paediatr 110(3):939-940

42. Solomon $\mathrm{IH}$, Normandin $\mathrm{E}$, Bhattacharyya $\mathrm{S}$, Mukerji SS, Keller K, Ali AS et al (2020) Neuropathological features of Covid-19. N Engl J Med 383(10):989-992

43. University of Liverpool (2021) COVID-19 drug interactions. https://covid19-druginteractions. org/.Zugegriffen:6. Juni 2021

44. Vohora D, Jain S, Tripathi M, Potschka H (2020) COVID-19 and seizures: is there a link? Epilepsia 61(9):1840-1853

45. Vollono C, Rollo E, Romozzi M, Frisullo G, ServideiS, Borghetti A et al (2020) Focal status epilepticus as 
unique clinical feature of COVID-19: a case report. Seizure 78:109-112

46. World Health Organisation (2021) Homepage. www.who.int.Zugegriffen: 16. Mai 2021

47. Xiong W, Mu J, Guo J, Lu L, Liu D, Luo J et al (2020) New onset neurologic events in people with COVID-19 in 3 regions in China. Neurology 95(11):e1479-e87

48. Zhu N, Zhang D, Wang W, Li X, Yang B, Song J et al (2020) A novel coronavirus from patients with pneumonia in China, 2019. N Engl J Med 382(8):727-733

49. Zimmermann P, Curtis N (2020) Coronavirus infections in children including COVID-19: an overview of the epidemiology, clinical features, diagnosis, treatment and prevention options in children. Pediatr InfectDis J 39(5):355-368

\section{Josef Zentner}

\section{Surgical Treatment of Epilepsies}

Diagnosis, Surgical Strategies, Results

\section{Cham, Springer Nature Switzerland 2020, 404 S., (ISBN: 978-3- 030-487474-8), Hardcover 171,19 € EUR, als Softcover und eBook 128,39 $€$}

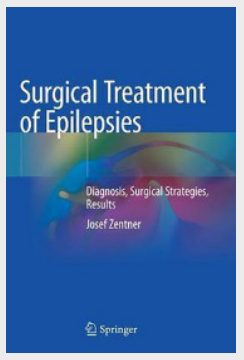

Eine aktuelle und umfassende Darstellung der Epilepsiechirurgie von einem der weltweit erfahrendsten Experten aus einer Hand hat es lange Zeit nicht mehr gegeben. Ein vor einigen Jahren im gleichen Verlag erschienenes Werk beschränkt sich auf operationstechnische Aspekte mit Fokus auf die Montreal-Schule (1). Das vorliegende Buch ist ein Beweis dafür, dass auch heute noch ein Einzelner eine umfassende Darstellung des Themas leisten kann! Man merkt es dem Text von Anfang bis Ende an, dass der Autor das "große Ganze" im Blick hatte und über eine jahrzehntelange Erfahrung in der resektiven Epilepsiechirurgie verfügt.

Josef Zentner, Jahrgang 1952, absolvierte seine Ausbildung an der Neurochirurgischen Universitätsklinik in Freiburg im Breisgau; das Buch ist seinem Lehrer Wolfang Seeger gewidmet, mit dem er 2002 bereits ein Buch publiziert hat (2). Von 1991 bis 1997 war Zentner Leitender Oberarzt und Vertreter von Johannes Schramm in der Bonner Neurochirurgie, bevor er bis 2018 als Direktor der Abteilung Allgemeine Neurochirurgie der Neurochirurgischen Universitätsklinik nach Freiburg zurückkehrte und insbesondere zusammen mit den Kollegen des Epilepsiezentrums Kehl-Kork und der Heidelberger Neuropädiatrie ein epilepsiechirurgisches Programm aufbaute. Von 2003 bis 2005 war er Vorsitzender unserer Fachgesellschaft; das Buch hat er nach seiner Emeritierung verfasst.

In 18 Kapiteln mit ausgeprägtem Reviewcharakter und einem jeweils vorangestellten Zitat wird ein umfassender Überblick zum Thema gegeben. Beginnend mit der Geschichte der Epilepsiechirurgie und der prächirurgischen Diagnostik liegt der Schwerpunkt auf der Darstellung der verschiedenen resektiven Verfahren einschließlich Komplikationen. Einen gebührenden Raum erhielt aber auch die nicht-resektive Epilepsiechirurgie. Außerdem wurden besondere Aspekte wie Langzeit-Epilepsie-assoziierte Tumoren, MRT-negative Epilepsien, Re-Operationen sowie die Neuropathologie berücksichtigt. Die drei letzten, kurzen Kapitel sind der Kosten-Nutzen-Frage, dem derzeitigen Stellenwert und einer persönlichen Betrachtung der Epilepsiechirurgie aus wissenschaftsphilosophischer Sicht gewidmet. Alle Kapite werden durch umfassende Literaturhinweise ergänzt. Als kurze Zugabe finden sich am Ende noch einige Seiten über Epilepsie im Spiegel der Künste. Bei einzelnen Kapiteln haben verschiedene, im Vorwort erwähnte Kolleginnen und Kollegen mit Hinweisen und beim Korrekturlesen geholfen, alleiniger Autor ist Josef Zentner.

Insgesamt handelt es sich um ein sehr gelungenes und sehr empfehlenswertes Buch, das für alle klinisch tätigen Kolleginnen und Kollegen in der Epileptologie interessant ist, die sich mit der Epilepsiechirurgie beschäftigen, sei es auf neurologischem, neuropädiatrischem, neurochirurgischem, neuropathologischem oder auch neuroradiologischem Fachgebiet. Christian Elger schreibt in seinem Vorwort "I read it and I will read it again". Auch wir haben das Buch seit seinem Erscheinen wiederholt als hervorragende Informationsquelle genutzt und sind uns sicher, auch in der Zukunft immer wieder darauf zurückzugreifen. Sollte es zu einem Nachdruck oder einer Neuauflage kommen, sollte unbedingt das bislang fehlende Stichwortverzeichnis ergänzt werden. Ausstattung und durchgehend vierfarbiger Druck sind SpringerStandard, der Preis ist akzeptabel.

1) Girvin JP. Operative Techniques in Epilepsy. Cham - Heidelberg - New York et al, Springer International Switzerland 2015

2) Seeger $W$, Zentner J. Neuronavigation and Neuroanatomy. Wien - New York, Springer 2002

Günter Krämer, Friedhelm Schmitt 